\title{
Diagnostic delay in a patient with reactional lepromatous leprosy treated as testicular tuberculosis*
}

\author{
Mayara Elisa Batista Ferreira ${ }^{1}$, Lucia Martins Diniz ${ }^{2}, K$ arla Spelta ${ }^{1}$, Elton Almeida Lucas ${ }^{3}$
}

DOI: http:/ / dx.doi.org/10.1590/abd1806-4841.20198327

\begin{abstract}
Leprosy is a chronic neglected and stigmatizing disease. Due to involvement of the peripheral nerves, it can result in physical disabilities, deformities, and emotional damage if not diagnosed and treated promptly. This is the case of a young patient with testicular pain and swelling and no improvement after a specific therapeutic scheme for tuberculosis. Clinical and laboratory reevaluation revealed hypoesthetic skin patches associated with post-burn crusted ulcers on the left arm, thickening of ulnar nerves, atrophy of interosseous muscles of the hands, positive skin smear microscopy, and testicular histopathology with numerous bacilli forming globi. These findings indicated lepromatous leprosy with type II reaction.
\end{abstract}

Keywords: Delayed diagnosis; Leprosy; Mycobacterium leprae

Leprosy is a chronic infectious disease that represents a serious public health problem, especially in developing countries. Due to neural damage, it can trigger physical deformities and disabilities. Early detection and treatment are essential to reduce morbidity in individuals affected by the disease. ${ }^{1,2}$

Herein is reported a case of a man with testicular pain and edema treated for tuberculosis during five months, with no clinical improvement. He was reevaluated and diagnosed with lepromatous leprosy in type II reaction.

The patient was 31 years old, exhibited testicular pain and swelling, concomitant with fever, night sweats, dyspnea, and malaise, and was submitted to urological treatment with oral antibiotics. Due to the absence of clinical improvement, the patient underwent testicular and epididymis biopsy. The results indicated chronic granulomatous inflammatory reaction and fibrosis, and positive acid-fast bacilli consistent with tuberculosis. Treatment was initiated with rifampin, isoniazid, pyrazinamide, and ethambutol administered until the fifth dose with no favorable response. The treatment was discontinued and the patient was referred to dermatology service. In addition to testicular edema, the patient exhibited crusted ulcers on the left arm and elbow, hypopigmented patches with scarce hairs and altered thermal/painful sensation in the left

\footnotetext{
Received 26 March 2018.

Accepted 19 June 2018

* Work conducted at the Hospital Universitário Cassiano Antonio Moraes, Universidade Federal do Espírito Santo, Vitória (ES), Brazil. Financial support: None.

Conflict of interest: None.

Dermatology Service, Universidade Federal do Espírito Santo, Vitória (ES), Brazil.

Department of Internal Medicine, Discipline of Dermatology, Universidade Federal do Espírito Santo, Vitória (ES), Brazil.

Discipline of Pathology, Universidade Federal do Espírito Santo, Vitória (ES), Brazil.

Mailing Address:

Mayara Elisa Batista Ferreira

E-mail: mayarabf@yahoo.com.br 
forearm, atrophy of interosseous muscles of the hands, thickening of ulnar nerves, bilateral madarosis, and infiltration in the ear pinnae (Figures 1, 2 and 3). It was concluded by the diagnosis of lepromatous leprosy in type II reaction with orchitis. The treatment included multibacillary multi-drug therapy and prednisone $1 \mathrm{mg} / \mathrm{kg} /$ day, a drug chosen to avoid serious sequelae, such as infertility. The slit-skin smear was positive (bacilloscopic index $=3.25$ with intact bacilli). The histopathological reassessment of the testicle indicated abundant acid-fast bacilli forming globi (Figure 4).

Leprosy is a challenge to public health, in terms of management and control programs. ${ }^{1,2}$ Early detection and treatment are essential for interrupting the transmission chain and preventing physical disabilities. ${ }^{3}$

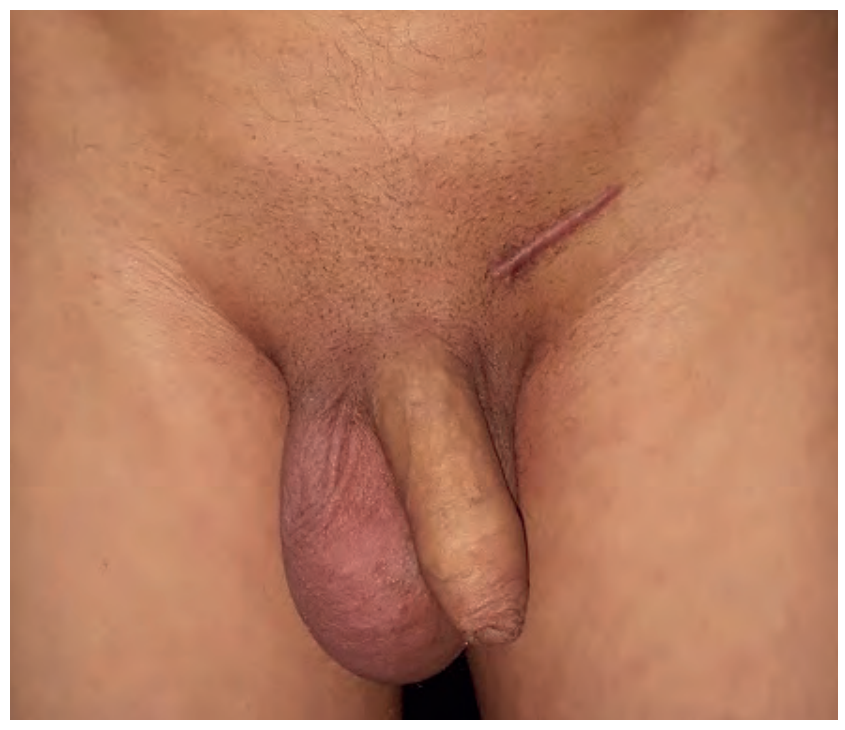

FIGURE 1: Scrotal erythema and testicular swelling

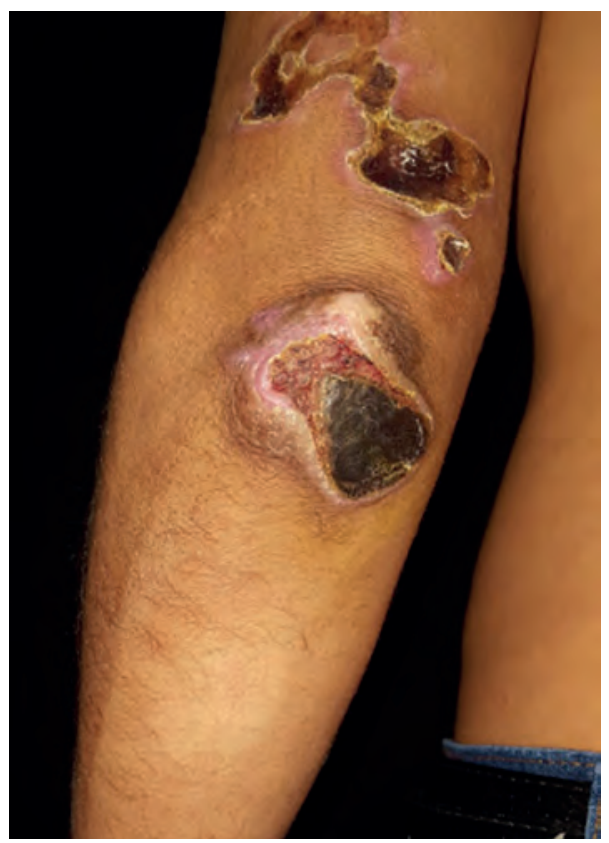

Figure 2: Extensive crusted ulcers in the left arm and elbow, after local hot water compress and mild hypochromic macule with scarce hairs in the forearm

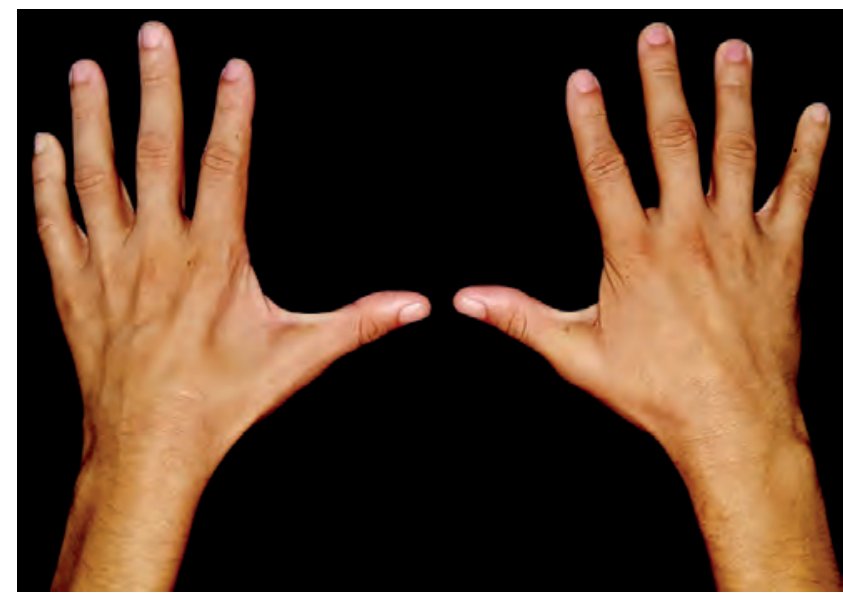

FigURE 3: Atrophy of interosseous muscles of the hands, especially left
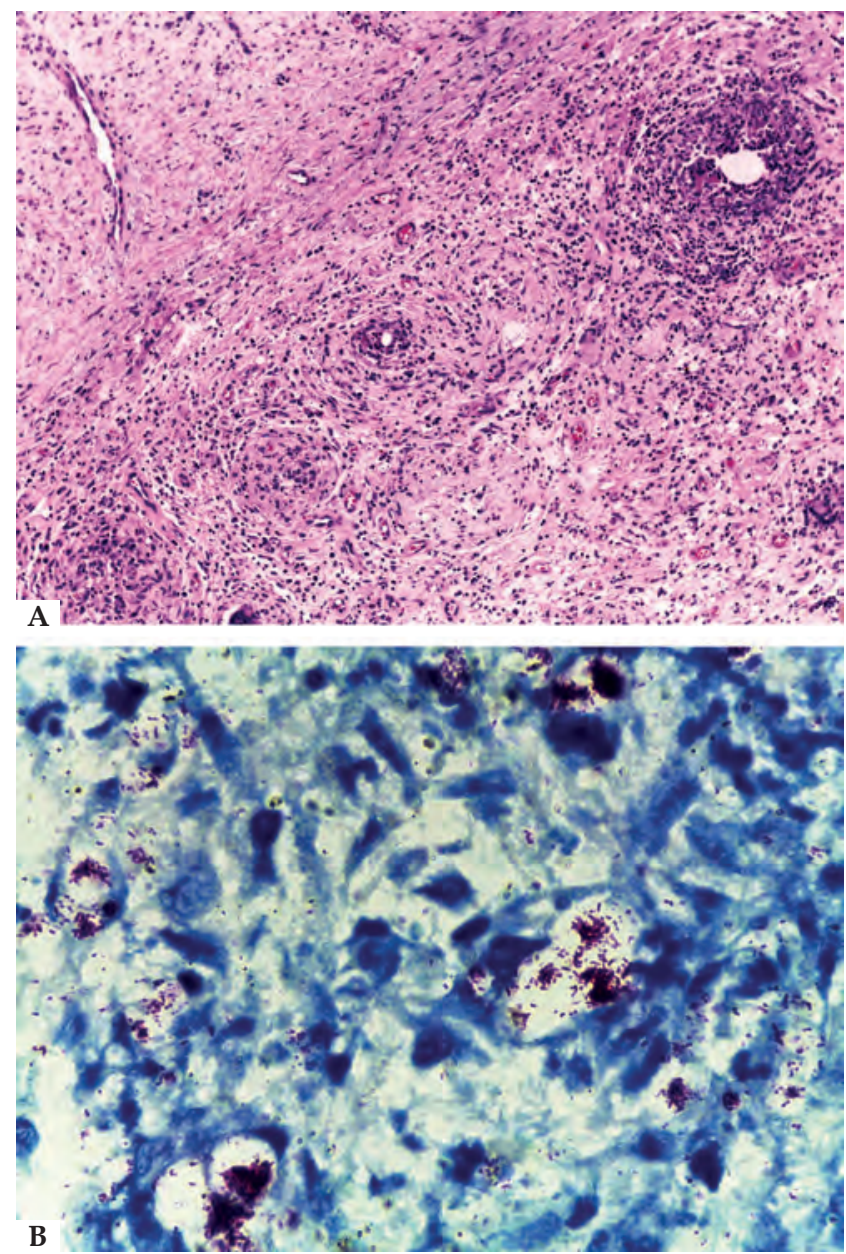

Figure 4: Testicular histopathology. A - Fibrotic conjunctive neoformation with replacement of the elements of testicular parenchyma, accompanied by mixed inflammatory infiltrate, with vacuolated macrophages and giant cells (Hematoxylin \& eosin, x40). B - Abundant acid-fast bacilli, some of which are clumped in globi (Ziehl-Neelsen, x100) 
Leprosy reactional states are the main cause of peripheral nerve damage, with great potential for causing physical disabilities and evolving into irreversible deformities. ${ }^{4}$ Besides orchitis, other extracutaneous manifestations may also occur in type II reactions, such as iridocyclitis, uveitis, lymph node enlargement, arthritis, synovitis, dactylitis, hepatitis, splenitis, laryngitis, and pharyngitis. ${ }^{1}$ These consequences can reduce working capacity and cause psychological damage, thus limiting social life, mainly among young individuals. ${ }^{4}$

This case demonstrates the importance of the patient's integral assessment, given that initial care had only focused on the external genitals, and not on the other signs and symptoms (madarosis, infiltration in the ear lobes, hypopigmented patch on the left arm, and atrophy of the interosseous muscles), which were typical of leprosy, as well as orchitis as a manifestation of the reactional process of the disease. The diagnosis and treatment were postponed, exposing the patient to possible sequelae and household contacts to transmission of the infection, thus perpetuating the chain of disease transmission.

Early diagnosis and treatment do not only prevent the progression of the disease; they also promote the elimination of the infection source. These are strategies used to control this endemic disease. ${ }^{4,5} \square$

\section{REFERENCES}

1. Talhari S, Penna GO, Gonçalves HS, de Oliveira MLW. Hanseníase. 5th ed. Manaus: DiLivros; 2015.

2. World Health Organization. Department of Control of Neglected Tropical Diseases. Global leprosy update, 2013; reducing disease bruden. Wkly Epidemiol Rec. 2014;89:839-400.

3. Lastória JC, Abreu MA. Brazilian Society of Dermatology against leprosy. An Bras Dermatol. 2016;91:393-5.
4. Brasil. Ministério da Saúde. Secretaria de Políticas de Saúde. Departamento de Atenção Básica. Guia para o controle da hanseníase. Brasília: Ministério da Saúde; 2002. (Série A. Normas e Manuais Técnicos; n. 111).

5. Noriega LF, Chiacchio ND, Noriega AF, Pereira GA, Vieira ML. Leprosy: ancient disease remains a public health problem nowadays. An Bras Dermatol. 2016;91:547-8.

\section{AUTHORS'CONTRIBUTIONS}

Mayara Elisa Batista Ferreira
Elaboration and writing of the manuscript; Obtaining, analyzing and interpreting the data; Critical review of the literature; Critical review of the manuscript
Lucia Martins Diniz
Approval of the final version of the manuscript; Elaboration and writing of the manuscript; Effective participation in research orientation; Critical review of the manuscript
Karla Spelta
Intellectual participation in propaedeutic and/or therapeutic conduct of the cases studied; Critical review of the manuscript
Elton Almeida Lucas
Approval of the final version of the manuscript; Obtaining, analyzing and interpreting the data

How to cite this article: Ferreira MEB, Diniz LM, Spelta K, Lucas EA. Diagnostic delay in a patient with reactional lepromatous leprosy treated as testicular tuberculosis. An Bras Dermatol. 2019;94(2): 236-8 